\title{
An exploration of family dynamics in adults who self-harm
}

\author{
Ruth Buckmaster ${ }^{1,2}$ (D) , Muireann McNulty ${ }^{1}$ and Suzanne Guerin ${ }^{1 * *}$ (D) \\ ${ }^{1}$ School of Psychology, University College Dublin, Dublin 4, Ireland \\ ${ }^{2}$ Health Service Executive Midlands, Laois/Offaly, Ireland
}

Objectives: The aim of the present study was to explore how adults who self-harm experience family relationships.

Methods: A phenomenological design was employed to examine the dynamic relationship between self-harm and family systems. Semi-structured interviews were conducted with six female adults who attend a community mental health service and engage in self-harm. Transcripts were analysed using Interpretative Phenomenological Analysis (IPA).

Results: Four superordinate themes emerged from the data and two subordinate themes emerged within each superordinate theme: family interactive patterns (subordinate themes: enmeshed patterns and culture of 'getting on with it'), searching for meaning (subordinate themes: expressing emotional turmoil and engrained worthlessness), relating to others (subordinate themes: guilt and feeling misunderstood) and journey towards life without self-harm (subordinate themes: acceptance and family support).

Conclusions: Findings emphasise the role of family systems in understanding self-harm in adults. The study highlights the need for family-based interventions for family members who support adults that self-harm.

Received 05 December 2020; Revised 26 May 2021; Accepted 10 August 2021

Key words: family dynamics, family relationships, family systems, interpretative phenomenological analysis, self-harm, self-harming behaviours, self-injury.

The 'function' of self-harm has been explored extensively in the literature, in an attempt to understand why individuals self-harm (Chapman et al. 2006; Nielsen et al. 2017; Stone \& Sias, 2003). Robust research evidence indicates that self-harm functions primarily as a means of altering negative emotional experiences (Nielsen et al. 2017). Therefore, self-harm is often viewed as a coping mechanism, to manage stressful experiences in the short term (Chapman et al. 2006; Stone \& Sias, 2003). Nielsen et al. (2017) investigated self-harm functions in an adult community sample and found that self-harm was associated with lower levels of approach (indirectly approaching a concern), reappraisal and emotion regulation coping, and higher levels of both avoidant coping and experiential avoidance. In terms of interpersonal functions, it has been suggested that self-harm serves to communicate distress, elicit social support, escape from undesired interpersonal situations or demands, assert autonomy, demonstrate strength, and to seek belonging or acceptance within a group (Klonsky, 2007).

A number of different theories have been reference to understand the relationship between self-harm and family dynamics. Many of these theories acknowledge an emotional regulation aspect of self-harm (Nock, 2009). Cognitive theorists conceptualise self-harm as occurring as a result of problem-solving deficits (Nezu

*Author for correspondence: Email suzanne.guerin@ucd.ie et al. 2010). They also suggest that those who self-harm have attentional biases which lead them to focus on the negative aspects of interpersonal relationships, resulting in greater reliance on coping skills which are usually maladaptive, such as self-harm (Guerry \& Prinstein, 2010). Behavioural theorists emphasise the relational role of self-harm and suggest that self-harm occurs in response to interpersonal triggers, as a method of emotional regulation (Gibson et al. 2014). Developmental and family theorists emphasise the role of insecure attachments and suggest that a lack of adaptive coping skills required to manage childhood trauma and current familial relationships can result in self-harm (Glazebrook et al. 2016).

A combined theoretical background of behavioural, developmental and family functioning models subscribes to an exploration of the dynamic interaction between self-harm and family relationships, as it draws on early family childhood experiences as well as current interpersonal functioning within the family. Despite the presence of many theories which emphasise the role of familial relationships in self-harm, this area has received relatively little research attention in the adult population (Levesque et al. 2010).

Research investigating self-harm in adolescents has suggested that the relationship between self-harm and family dysfunction is a dynamic one, whereby an adolescent in a vulnerable family system uses self-harm as a maladaptive coping strategy, which adds further stress 
to the family system (Morgan et al. 2013). A narrative review of 126 studies which examined family factors associated with self-harm in the adolescent population highlighted a range of strong, and mostly negative emotive reactions by parents when learning about their adolescents' self-harm, such as distress, worry, shame and a sense of responsibetility (Fortune et al. 2016). Adolescent self-harm was found to be associated with poor attachment and family cohesion, low parental support, warmth and adaptability. Based on their findings, the authors recommended that therapeutic interventions for this cohort should be family orientated and should focus on ameliorating these familial difficulties.

Less empirical research exists in the field of adult self-harm and family relationships. However, findings from a systematic review of family relationships for adults who self-harm (Buckmaster et al. 2019) identified 27 studies which explored this phenomenon. This review indicated that insecure attachment to parents and romantic partners is associated with self-harm. In accordance with cognitive models, this relationship was found to be mediated by problem-focused coping, problem-solving abilities and stress. In terms of parenting, low parental care, overprotection, disempowering and rejecting parenting styles were positively associated with self-harm (Hahm et al. 2014), whereas supportive, open and accepting parenting styles were negatively associated with self-harm (Benau et al. 2017). Consistent with both developmental and interpersonal models, most studies in the review reported that sexual, emotional and physical abuse, perpetrated by both parents and romantic partners, and neglect were all associated with self-harm, although one study reported no relationship between abuse and parental neglect (Johnstone et al. 2016). The relationship between self-harm, and emotional abuse and neglect, was found to be mediated by pessimistic explanatory styles (a habitual interpretation of negative events as being stable and global) and academic self-efficacy, also showing evidence for the cognitive models of self-harm (Buser \& Hackney, 2012; Buser et al. 2015). Finally, consistent with Olson's (1999) theory of family functioning, cohesion and flexibility were found to be negatively associated with self-harm. The limited literature in family systems and the adult self-harming population highlights family dysfunction (Halstead et al. 2014), romantic relationship difficulties (Townsend et al. 2015) and attachment difficulties (Levesque et al. 2010) as predictors of self-harm.

The objective of the present study was to build on the scarce existing literature in this area and explore the lived experience of family dynamics in adults who engage in self-harm. This study aimed to explore the meaning-making experiences of adults who self-harm.

\section{Methodology}

Design

As there is little research in the self-harm literature that has described adults' experiences of family dynamics, an exploratory approach was chosen. A phenomenological framework was employed in order to capture participants' lived experiences of self-harm and its relationship with family systems. A phenomenological framework allows a detailed exploration of perceptions and understandings of individual's unique experiences, rather than identifying family relationships as being 'positive' or 'negative' influences on self-harming behaviours (Smith \& Osburn, 2015). It also allows for recognition of the dynamic nature of the relationships between individuals' self-harm and their family systems.

In accordance with the phenomenological design of the study, an interpretative phenomenological analysis (IPA) framework, as outlined by Smith et al. (2009) was used. IPA was developed from hermeneutic phenomenology that is both descriptive and interpretive. Although the focus of the research is the participant's meaning-making process, it recognises that the documentation of the participant's account cannot be independent of the interpretative role of the researcher. IPA involves a two-step process in which the participant attempts to make sense of their lived experience, and the researcher interprets their account (Smith et al. 2009).

\section{Participants and sampling}

Participants were six individuals who engaged in selfharm and who attend a community mental health service (age range 22-40 years; $M=27.8, S D=7.08$ ). Inclusion criteria were individuals who had completed an act of self-harm, in accordance with the following definition of self-harm: 'a non-fatal act in which an individual deliberately intended to cause self-harm through injury, ingestion of a substance in excess of the prescribed or therapeutic dose, ingestion of an illicit/recreational drug that was an act the individual regarded as self-harm or ingestion of a non-ingestible substance or object' (Hawton et al. 2000, p. 48). Exclusion criteria included individuals who are actively suicidal, currently experiencing symptoms of psychosis or currently on an inpatient psychiatric ward. Although male and female participants were eligible to take part in the study, only female participants opted in. The sample size of six participants was directed by the IPA analysis being used. A distinctive feature of IPA is a commitment to detailed analysis using a small sample size (Smith \& Osborn, 2015). Smith et al. (2009) have suggested that a sample size of three to six participants 
Table 1. Participant demographics

\begin{tabular}{|c|c|c|c|}
\hline Participant & Pseudonym & $\begin{array}{c}\text { Age category } \\
\text { (years) }\end{array}$ & $\begin{array}{l}\text { Method of } \\
\text { self-harm }\end{array}$ \\
\hline 1 & Julie & $26-30$ & Self-injury \\
\hline 2 & Catherine & $31-35$ & Self-injury \\
\hline 3 & Anna & $36-40$ & $\begin{array}{l}\text { Self-injury, inges- } \\
\text { tion of } \\
\text { substances, } \\
\text { purging }\end{array}$ \\
\hline 4 & Sarah & $21-25$ & Self-injury \\
\hline 5 & Emma & $21-25$ & Self-injury \\
\hline 6 & Laura & $21-25$ & Self-injury \\
\hline
\end{tabular}

is reasonable for an IPA study. Participants' demographics are presented in Table 1. In order to ensure anonymity, the participants were assigned the following pseudonyms: Anna, Catherine, Julie, Sarah, Emma and Laura. Furthermore, any names of people, places or organisations mentioned were also changed or omitted.

\section{Procedure}

Participants were recruited through a community mental health service by their clinicians. Purposive sampling and rolling recruitment were used in line with the IPA approach (Smith, 1996). Five participants were identified who met the aforementioned criteria through discussions with clinicians in the service and provided informed consented to take part in the study. There were no other participants identified by the clinicians in the service at this time and recruitment was suspended and the interviewing, analysis and write-up stages of the study began. When the analysis was completed, the researcher was contacted by a clinician to inform them of an additional potential participant who was interested in the study. This sixth participant's data were added and analysed at this point.

A semi-structured interview schedule was devised based on previous literature, relevant theories and the researchers' clinical experience. The first researcher conducted each of the six semi-structured interviews, which were audio-recorded and later transcribed verbatim by a research assistant. Interviews ranged from 35 to 63 minutes in duration $(M=52, S D=11.06)$.

\section{Data analysis}

IPA was completed in line with the protocol developed by Smith et al. (2009). The steps of analysis are outlined in Table 2. The first author analysed each of the transcripts. The second researcher also analysed the first transcript and themes were discussed between the
Table 2. Steps of IPA analysis as outlined by Smith et al. (2009)

1. Reading and noting of initial areas of interest and potential preconceptions.

2. Line by line analysis of semantic, linguistic and conceptual content.

3. Development of emerging theme.

4. Searching for connections across emerging themes within the transcripts of each participant.

5. Searching for patterns across the emerging themes of each participant.

6. Taking the analysis to a deeper level of interpretation by focusing on what the content of participants accounts might mean for them.

7. Grouping of superordinate and subordinate themes

8. Inclusion of quotes for each theme to ensure the themes relates accurately back to the data

9. Count of reoccurring themes to ensure validity of analysis

two researchers in order to ensure credibility in accordance with Elliott et al. (1999) guidelines for best practice in qualitative research. As the sixth participant's data were added to the analysis at a later stage, the third researcher who was not involved in the earlier analysis independently analysed the transcript, in addition to the analysis by the first author. This process was completed to allow for a reflection on the interpretation of the final participant and to prevent bias and preconceptions from the prior analysis of the first five participants impacting on the analysis of the sixth participant. Themes were reviewed in light of rereading of the transcripts; those which were not rich in evidence from the text were discarded. Themes were only included if there was evidence of the theme in at least half of the sample as recommended by Smith et al. (2009).

\section{Results}

The analysis of the six participants' accounts produced four superordinate themes: family interactive patterns, searching for meaning, relating to others and a journey towards life without self-harm. The subordinate themes within each of the four superordinate themes are presented in Table 3.

\section{Family interactive patterns}

All of the participants described experiencing unhelpful family interactive patterns. These unhelpful patterns were often quoted as triggers for participants' selfharming behaviours. Some participants referenced overinvolved family systems, while others commented on disengaged patterns, in which family difficulties were not addressed, and a pattern of 'getting on with it' was prevalent. 
Table 3. Table of superordinate and subordinate themes

\begin{tabular}{|c|c|}
\hline Superordinate theme & Subordinate theme \\
\hline $\begin{array}{l}\text { 1. Family interactive } \\
\text { patterns }\end{array}$ & $\begin{array}{l}\text { - Enmeshed patterns } \\
\text { - Culture of 'getting on with it' }\end{array}$ \\
\hline 2. Searching for meaning & $\begin{array}{l}\text { - Expressing emotional turmoil } \\
\text { - Engrained worthlessness }\end{array}$ \\
\hline 3. Relating to others & $\begin{array}{l}\text { - Guilt } \\
\text { - Feeling misunderstood }\end{array}$ \\
\hline $\begin{array}{l}\text { 4. Journey towards life } \\
\text { without self-harm }\end{array}$ & $\begin{array}{l}\text { - Acceptance } \\
\text { - Family support }\end{array}$ \\
\hline
\end{tabular}

\section{Enmeshed patterns}

Many of the participants described an enmeshed pattern in their family. Participants described these patterns as leading to a sense of feeling overwhelmed and out of control. Catherine noted that when she became pregnant she experienced fear and frustration at the prospect of her parents' level of involvement with her child's life. She described feeling a lack of control of in her birth giving process: 'I felt like I was afraid to go into labour because they'd know I was in labour and they'd try to take over'.

Laura demonstrated her reliance on her mother, saying that she 'can't sleep when mammy's not at home' and Emma described her mother as a 'mama bear' who protected her family at all costs. While there was an acknowledgement of her mother's good intentions, Emma also identified with feeling overwhelmed by her level of involvement. Similarly, Julie described her family unit as being 'like a mafia', depicting a group mentality in which individual opinions were not accepted. While Catherine and Emma described feelings of frustration in relation to this style and referenced it as a trigger for self-harm, Julie appeared to find comfort in this pattern and perceived it to be protective.

\section{Culture of 'getting on with it'}

All six participants described a family culture of silence surrounding issues such as mental health, abuse or other difficulties. Anna described feeling a need to be perceived as 'strong' and to not let difficulties affect her. Similarly, Laura noted that she would not let anyone see 'the vulnerable side'. Catherine described trying to break the silence and talk to her parents about her difficulties. She stated that when she told her parents she had been abused they had a dismissive response: 'sure that happens to everyone'. Sarah also suffered abuse from a family member as a child, and described receiving a similarly disregarding reaction from her parents when she told them: 'Oh, I was just told not to tell anyone else basically'.

\section{Searching for meaning}

All participants described searching for meaning in their self-harm and trying to make sense of why they engage in this behaviour. Self-expression was a prominent feature in the narratives surrounding meaning of all six participants. They described the difficulty they experience in managing their challenging emotions and searching for a way to express this. Another meaning which some participants conveyed was a feeling of being worthless. They described feeling like they were not worthy of others' love and that their bodies should show this.

\section{Expressing emotional turmoil}

Many participants identified their self-harm as being a way to express negative emotions and noted how the behaviour 'releases a little bit of tension'. In a family culture where verbal expression was not nurtured, participants spoke about trying to find a way to express the emotional turmoil they were experiencing: 'Well I suppose if you can't express yourself, you know, it's eh...you're going to suffer or you're going to do something to compensate you know?' Similarly, Catherine spoke about how she wanted her 'body to match [her] mind'. Participants also described an addictive aspect of this method of self-expression. Expressing oneself in this way became 'like a compulsion' or 'the very same as a heroin or drink addiction' for some participants.

\section{Engrained worthlessness}

Some participants made sense of their self-harm in terms of their self-worth. They felt unworthy of having healthy, unharmed bodies. Catherine described an inner conflict in that she had some sense that she should be trying to protect her body; however, her desire was to do the opposite: 'liking my body to a house being burgled but instead of wanting to reinforce it or make it stronger, I want to demolish it'. Other participants highlighted their struggle with accepting help when it was offered. They appeared to have internalised the culture of 'getting on with it'. When help was offered participants described feeling like this 'wasn't right' and 'there's no point in saying anything', as though they weren't worthy of others' love and help.

\section{Relating to others}

Participants' reports of the impact of self-harm on their relationships with family members varied. For some participants, self-harm was seen as having a significant effect on both their families and their relationships 
within their families, while others struggled to understand why their families felt affected by their self-harm. Two participants noted that their families were unaware of their self-harm and described how they were reluctant to tell them for fear of causing concern or emotional pain. For those participants, feelings of guilt were elicited. Other participants who did not see their self-harm as impacting on their family members reported feelings of frustration when relating to their family members. They described feeling misunderstood and unable to communicate openly and honestly with their families.

\section{Guilt}

Some participants identified their self-harming behaviours as being related to feelings of upset and worry for their family members and expressed feelings of guilt in relation to this. Anna highlighted that even though her son was too young to understand the behaviour that he had some awareness that she was suffering: 'He keeps crying at 12 o'clock, he wakes up at 12 o'clock in the night'. He says 'mammy, you're going to die on me'. Anna was tearful when describing the impact her self-harming behaviours were having on her children and stated that 'the roles are wrong', in relation to her children caring and worrying about her. She appeared to experience guilt and frustration when drawing links between the emotional burden she felt as a child when caring for her own mother, and her acceptance of help from her own children now. For other participants, their awareness of the impact of their self-harm on their family members was more vague 'I don't realise, you know, the seriousness it has in it for everyone else'.

\section{Feeling misunderstood}

Most participants suggested that their loved ones struggled to understand their self-harm. Julie highlighted her parents' openness around their lack of understanding, she says 'myself and your father don't understand it because we've come from a different era. You know, everything was swept under the carpet, you got on with life and that was it'. Julie appeared to meet this confusion and lack of awareness from her family with some level of inner conflict. She appeared to be comforted by their honesty in relation to their lack of understanding while also maintaining feelings of frustration and loneliness. Catherine also highlighted her frustration with her family's lack of understanding and how it prevented her from being open and honest with them. She spoke about a period of time when she was engaging in a self-harm and her family would say 'but you just got married?' Catherine appeared to feel misunderstood and described how her inability to enjoy the early stages of her marriage was a sign of how low her mood was at the time, which was something that her family could not understand.

\section{Journey towards a life without self-harm}

Most participants spoke about a desire to stop selfharming, and the strategies used to help them achieve a life without self-harm. All participants attended a community mental health service and two of the six participants also attended a support group for anxiety and depression. These help-seeking behaviours were described by participants as promoting acceptance and self-reflection, and in turn reducing self-harming behaviours. Despite the struggles with family members discussed above, family support was also a commonly referenced factor that protected participants from the urge to self-harm.

\section{Acceptance and self-reflection}

Many participants spoke about acceptance as a contributing factor in their journey towards a life without selfharm. Anna noted that she found comfort in recognising her mother's flaws and accepting her as she is: 'I was trying to get her to be the mother I wanted her to be, but she's never going to be the mother I want her to be'. Anna reflected on her comfort and feelings of contentment when she came to this realisation. She reported that she has begun to work on her 'own child inside' and that she has come to realise that she finds it more helpful to turn to members outside her family for support. Julie, Sarah and Laura also spoke about their preference to speak about their difficulties to individuals outside of their family and to professionals when in need of support, and how this has helped them to gain self-awareness and acceptance which in turn has helped to reduce their self-harming behaviours.

\section{Family support}

Although not all participants reported a desire to speak about their self-harm with family members, they all referenced the value and impact that family support has had on their journey towards life without self-harm. Three of six participants spoke about finding romantic partners who demonstrated unconditional love and support, which was in contrast to their experience of a lack of support they felt growing up in their biological families. Catherine recounted her pleasant surprise at her husband's reaction to an episode of self-harm, while she expected him to be angry and upset, instead saying 'let's go for a longer streak next time'.

Many participants appeared to be in the process of creating new family cultures of acceptance, openness and honesty both with their romantic partners, but also their children. Anna spoke about how she allowed her 
children to curse or punch pillows to encourage them to express themselves. When criticised by her mother, Anna responded 'No, it's not Mam. That's not terrible behaviour, he's expressing himself'. Many participants displayed hope, strength and courage in their stories. Despite challenging family dynamics and significant episodes of self-harm, many demonstrated selfawareness of previous problematic patterns and were optimistic about their hopes and plans for more adaptive and supportive patterns in the future.

\section{Discussion}

The aim of the present study was to explore the lived experience of family dynamics in an adult population who engage in self-harm. Semi-structured interviews were conducted with six individuals recruited though an adult community mental health service. A number of themes emerged in the data that provided a rich account of the dynamic relationship between family systems and self-harming behaviours. Findings from the present study produced both convergences and divergences from previous literature in the area of adult self-harm. Difficulties in family relationships were characterised by disengaged and enmeshed patterns, the function of self-harm in this context, and the confusion and associated guilt which arose as a result were described. Findings were also observed in terms of hopefulness, protective family patterns and the determination of individuals to develop functional family relationships.

The family interactive patterns described by participants in the present study are consistent with theories of dysfunctional patterns of family dynamics described by Olson (1999). Enmeshed patterns depicted by participants are reflective of the extreme end of the cohesion scale, whereas the culture of 'getting on with it' could be interpreted as a disengaged pattern. These dysfunctional patterns were described as triggers for selfharming behaviours, which is also consistent with previous research in the area of adult self-harm (Halstead et al. 2014). The 'culture of getting on with it' theme is also consistent with theories of interpersonal and developmental models (Nock, 2009) and is in line with previous research describing disengaged parenting styles (Hahm et al. 2014).

Participants' acknowledgement of their families' confusion in relation to self-harm in the present study has also been previously documented in Lindgren et al. (2010) account of parents' reactions to their adult daughters' self-harm. The guilt expressed by participants was predominantly related to their awareness of the impact of their self-harm on their family members, which has also been previously documented in the literature (Wadman et al., 2016). In particular, role reversal in the adult-child relationship was noted as a trigger for feelings of guilt. Previous research by Wu et al. (2007) documented the misplaced parentchild subsystem which can occur in the context of parents who self-harm. The guilt aspect of the phenomenon was not reported in Wu et al.'s study (2007), although this study documented the experiences of the adult children of parents who self-harm, rather than the adults themselves who self-harm, as was the case in the present study.

Insight was gained in terms of the function of self-harm in the context of family relationships. The meaning attributed to their self-harm related to the expression of emotional turmoil and the engrained worthlessness which they felt. Self-harm as a method of self-expression, stress release and self-soothing has been widely documented in the self-harm literature (Nock, 2010). However, findings from the present study suggest that a potential reason for the need for this form of self-expression is being part of a family who struggle to verbalise or express difficulties otherwise. The addictive nature of self-harm in this regard was also described, which has also been described previously in the literature (Brown \& Kimball, 2013).

Participants in the present study offered a sense of hopefulness and optimism in their plans for the future. Previous research exploring the relationship between self-harm and romantic relationships have suggested that relationship difficulties (Townsend et al. 2015) and abandonment over attachment (Levesque et al. 2010) were significant predictors of self-harm in adults. However, in the present study, participants reported healthy relationships with romantic partners, which were viewed as a protective factor for self-harming behaviours. Participants described how interpersonal difficulties with other members of their family, and cultures of not speaking about difficulties had motivated them to develop and nurture open and honest communication with their romantic partners. They highlighted the positive impact of this communicative pattern on their self-harm and described feelings of hope and optimism that they would lead lives free of self-harm in the future.

\section{Strengths and limitations}

A phenomenological design was deemed appropriate to address the present research question due to the complexity of the issue and the lack of previous research in the area (Smith et al. 2009). There are both strengths and limitations of this approach which should be considered. An IPA approach gave depth and richness to the personal accounts and internal meaning-making processes of this small sample of participants experiencing a specific phenomenon. However, it cannot be 
assumed that the findings of the present study apply to other self-harming adults. Rather, it offers a foundation for more research to be conducted and begins to develop our understanding of what it might be like to be a self-harming adult. Similarly, the small selective sample of 6 participants could be considered a limitation of the study. While this sample size is in line with Smith and Osburn (2015) recommendation of sample size for IPA studies, the findings of six participants cannot be generalised to the population of adults who self-harm.

Of the six participants in the study, five presented with cutting only, while the sixth reported multiple methods of self-harm. It could be argued that the sixth participant was heterogeneous to the other five participants. However, arguments have been made in the literature for the use of broader definitions of self-harm (Turp, 2002) in response to the clinical acknowledgement of the prevalence of multiple forms of self-harm. Studies have reported no clinical differences between individuals who engage in self-injury compared to individuals who engage in other methods of self-harm (Hooley \& Germain, 2014).

The interpretative facet of IPA must also be considered. Every effort was made in the analysis to ensure that the themes reflected the psychological processes of the participants, by referring back to participants' previous passages and attempting to avoid the use of the author's previous knowledge of theoretical frameworks, as recommend by Smith (2004). However, it is noteworthy that the clinical experience and awareness of theoretical underpinnings may have unknowingly influenced the analysis of the transcripts. In order to control for bias in this regard, a reflexive diary was kept throughout the analysis process, the second researcher analysed one of the participant's transcript and the third reviewer analysed a different participant's transcript.

\section{Clinical implications}

Although the findings of the present study are not generalisable to the adult self-harming population as a whole, they highlight the value of exploring family relationships when conceptualising and treating selfharming behaviour in adults. Due to the small sample size of the current study, it was deemed more appropriate to offer suggestions for areas for exploration when working with adults who self-harm, rather than making recommendations for specific therapeutic models.

Exploring an adult's patterns of interaction with their family members (including romantic partners) may be of benefit in a therapeutic intervention. Self-harm harm was found in the present study to be associated with both enmeshed and disengaged patterns. Identifying if a family is disengaged, enmeshed, rigid or chaotic could guide the therapist in training the adult or family in the skills necessary to reach an equilibrium on cohesion spectrums, with the aim of reducing self-harm.

It is recommended that clinicians explore the meaning of self-harm for the individual. Self-harm may be a method of expressing emotion turmoil where other forms of expression are not encouraged. Where this is the case, therapeutic models which help clients to express their difficult emotions in an adaptive way may be of benefit. Building a client's understanding of their self-harm and helping them to find a way to communicate this with their family members (where appropriate) may help to improve the family's understanding of the behaviour and alleviate feelings of guilt for the client. Psychoeducation about the function of emotions and value of emotional expression, as well as family work to coach family members in adaptive emotional experiencing, may be helpful for some members of the family.

In the present study, participants were found to identify with feelings of hope, resilience and a desire to live a life without self-harm. In the family context, it may be beneficial to help clients to make sense of their family dynamics to identify what needs are met in these relationships, which relationship can change and which cannot; therapeutic models which allow for family difficulties to be processed outside of the family may be helpful in this context. Clients may also benefit from exploring their desires in relation to family cultures and being supported to find a way to achieve such dynamics.

\section{Conclusion}

The present study employed a phenomenological design to explore the lived experiences of family dynamics in the adult self-harm population. IPA facilitated the acknowledgement of the dynamic and complex relationship between self-harm and family systems in the adult population. Four superordinate themes emerged within the data; family interactive patterns, searching for meaning, relating to others and journey towards a life without self-harm. Findings emphasise the need for the recognition of role of family systems in interventions for those who self-harm.

\section{Acknowledgements}

The authors would like to acknowledge the generosity of the participants in giving their time and sharing their experiences for the purposes of this research.

\section{Conflict of interest}

There are no conflicts of interest to report. 


\section{Ethical Standards Statement}

Ethical approval was obtained by the relevant university and organisational committees. Ethical approval obtained included approval for the publication of the study's findings. The authors assert that all procedures contributing to this work comply with the ethical standards of the relevant national and institutional committee on human experimentation with the Helsinki Declaration of 1975, as revised in 2008. This project was approved by the National Forensic Mental Health Service Research and Ethics Committee.

\section{Financial Support}

No specific funding was provided for this project. The first author completed this piece of research as part of a clinical doctorate programme. The second and third authors supervised this project as a component of their role at the university.

\section{References}

Benau EM, Jenkins AL, Conner BT (2017). Perceived parental monitoring and sexual orientation moderate lifetime acts of non-suicidal self-injury. Archives of Suicide Research 21, 322-340. DOI: 10.1080/13811118.2016. 1182092.

Brown TB, Kimball T (2013). Cutting to live: a phenomenology of self-harm. Journal of Marital and Family Therapy 39, 195-208. DOI: 10.1111/j.1752-0606.2011. 00270.x.

Buckmaster R, McNulty M, Guerin S (2019). Family factors associated with self-harm in adults: a systematic review. Journal of Family Therapy 41, 537-558.

Buser T, Hackney H (2012). Explanatory style as a mediator between childhood emotional abuse and nonsuicidal selfinjury. Journal of Mental Health Counseling 34, 154-169.

Buser TJ, Peterson CH, Kearney A (2015). Self-efficacy pathways between relational aggression and nonsuicidal self-injury. Journal of College Counseling 18, 195-208.

Chapman AL, Gratz KL, Brown MZ (2006). Solving the puzzle of deliberate self-harm: the experiential avoidance model. Behaviour Research and Therapy 44, 371-394. DOI: 10.1016/j.brat.2005.03.005.

Elliott R, Fischer CT, Rennie DL (1999). Evolving guidelines for publication of qualitative research studies in psychology and related fields. British Journal of Clinical Psychology 38, 215-229. DOI: 10.1348/014466599162782.

Fortune S, Cottrell D, Fife S (2016). Family factors associated with adolescent self-harm: a narrative review. Journal of Family Therapy 38, 226-256. DOI: 10.1111/14676427.12119 .

Gibson J, Booth R, Davenport J, Keogh K, Owens T (2014) Dialectical behaviour therapy-informed skills training for deliberate self-harm: a controlled trial with 3-month follow-up data. Behaviour Research and Therapy 60, 8-14. DOI: 10.1016/j.brat.2014.06.007.
Glazebrook K, Townsend E, Sayal K (2016). Do coping strategies mediate the relationship between parental attachment and self-harm in young people? Archives of Suicide Research 20, 205-218. DOI: 10.1080/13811118.2015. 1004495.

Guerry JD, Prinstein MJ (2010). Longitudinal prediction of adolescent nonsuicidal self-injury: examination of a cognitive vulnerability-stress model. Journal of Clinical Child and Adolescent Psychology 39, 77-89.

Hahm HC, Gonyea JG, Chiao C, Koritsanszky LA (2014). Fractured identity: a framework for understanding young Asian American women's self-harm and suicidal behaviors. Race and Social Problems 6, 56-68. DOI: 10. 1007/s12552-014-9115-4.

Halstead RO, Pavkov TW, Hecker LL, Seliner MM (2014). Family dynamics and self-injury behaviors: a correlation analysis. Journal of Marital and Family Therapy 40, 246-259. DOI: 10.1111/j.1752-0606.2012.00336.x.

Hawton K, Fagg J, Simkin S, Bale E, Bond A (2000). Deliberate self-harm in adolescents in Oxford, 1985-1995. Journal of Adolescence 23, 47-55. DOI: 10.1006/jado. 1999.0290.

Hooley JM, St Germain SA (2014). Should we expand the conceptualization of self-injurious behavior? Rationale, review, and recommendations. In The Oxford Handbook of Suicide and Self-injury (ed. M. K. Nock), pp. 47-60. Oxford University Press: New York.

Johnstone JM, Carter JD, Luty SE, Mulder RT, Frampton CM, Joyce PR (2016). Childhood predictors of lifetime suicide attempts and non-suicidal self-injury in depressed adults. Australian \& New Zealand Journal of Psychiatry 50, 135-144. DOI: 10.1177/ 0004867415585581.

Klonsky ED (2007). The functions of deliberate self-injury: a review of the evidence. Clinical Psychology Review 27, 226-239. DOI: 10.1016/j.cpr.2006.08.002.

Levesque C, Lafontaine MF, Bureau JF, Cloutier P, Dandurand C (2010). The influence of romantic attachment and intimate partner violence on nonsuicidal self-injury in young adults. Journal of Youth and Adolescence 39, 474-483. DOI: 10.1007/s10964-009-9471-3.

Lindgren BM, Åström S, Graneheim UH (2010). Held to ransom: parents of self-harming adults describe their lived experience of professional care and caregivers. International Journal of Qualitative Studies on Health and Well-being 5, 5482. DOI: 10.3402/qhw. v5i3.5482.

Morgan S, Rickard E, Noone M, Boylan C, Carthy A, Crowley S, Butler J, Guerin S, Fitzpatrick C (2013). Parents of young people with self-harm or suicidal behaviour who seek help-a psychosocial profile. Child and Adolescent Psychiatry and Mental Health 7, 13. DOI: 10. 1186/1753-2000-7-13

Nezu AM, Nezu CM, D'Zurilla TJ (2010). Problem-solving therapy. In Cognitive and Behavioral Theories in Clinical Practice (ed. N. Kazantzis, M. A. Reinecke \& A. Freeman), pp. 76-114. Guilford Press: New York.

Nielsen E, Sayal K, Townsend E (2017). Dealing with difficult days: functional coping dynamics in self-harm 
ideation and enactment. Journal of Affective Disorders 208, 330-337. DOI: 10.1016/j.jad.2016.08.036.

Nock MK (2009). Understanding non-suicidal self-injury. American Psychological Association: Washington, DC

Nock MK (2010). Self-injury. Annual Review of Clinical Psychology 6, 339-363. DOI: 10.1146/annurev.clinpsy. 121208.131258.

Olson D (1999). Circumplex model of marital and family systems. Journal of Family Therapy 22, 144-167. DOI: 10. 1111/jftr.12331.

Smith JA (1996). Beyond the divide between cognition and discourse using interpretative phenomenological analysis in health psychology. Psychology and Health 11, 261-271. DOI: $10.1080 / 08870449608400256$.

Smith JA (2004). Reflecting on the development of interpretative phenomenological analysis and it's contribution to qualitative research in psychology. Qualitative Research in Psychology 1, 39-54. DOI: 10.1191/ 1478088704qp004oa.

Smith JA, Flowers P, Larkin M (2009). Interpretative Phenomenological Analysis: Theory, Method and Research. Sage: London.

Smith JA, Osburn M (2015). Interpretative phenomenological analysis. In Qualitative Psychology:
A Practical Guide to Methods (ed. J. A. Smith) pp. 51-80. Sage: London.

Stone JA, Sias SM (2003). Self-injurious behavior: a bi-modal treatment approach to working with adolescent females. Journal of Mental Health Counseling 25, 112-125. DOI: 10.17744/mehc.25.2. gjk49quemhvna1dq.

Townsend E, Ness J, Waters K, Kapur N, Turnbull P, Cooper J, Bergen H, Hawton K (2015). Self-harm and life problems: findings from the Multicentre Study of Self-harm in England. Social Psychiatry and Psychiatric Epidemiology 1-10. DOI: 10.1007/s00127-0151136-9.

Turp M (2002). The many faces of self-harm. Psychodynamic Practice 8, 197-217. DOI: 10.1080/135333330210154655.

Wadman R, Clarke D, Sayal K, Vostanis P, Armstrong M, Harroe C, Majumder P, Townsend E (2016). An interpretative phenomenological analysis of the experience of self-harm repetition and recovery in young adults. Journal of Health Psychology 22, 1631-1641. DOI: 10. 1177/1359105316631405.

Wu HC, Lin MF, Yu SH (2007). Self-harm vs. harming others: the lived experiences of a dysfunctional family. $H u$ Li Za Zhi 54, 56-63. 